\title{
The Editing method of "Electronic patients" based on XML DataBase and Relational DataBase
}

\author{
Kui ZHOU ${ }^{1, a}$,Hongna $\mathrm{LI}^{2, \mathrm{~b}}$ \\ ${ }^{1}$ Maritime College, Tianjin University of Technology, Tianjin 300384, China \\ ${ }^{2}$ Maritime College, Tianjin University of Technology, Tianjin 300384, China \\ aemail: 2289970487@qq.com , bemail:lihongna2005@126.com
}

Keywords: Procedure opening method; First aid teaching; XML database

\begin{abstract}
The design of the data storage abandons the abuse of traditional database low query efficiency 、 not easy to maintain、 long development cycle of disadvantage, by combining XML and relational databases for data storage, satisfies the characteristics of the editor's information quantity and high speed running of the electronic patients. In the software module, the modular development method of the transplantation is adopted, "Electronic patients" case editing module can be ported to different medical teaching or rescue system software which. It provides a software which can effectively solve the contradiction between efficiency and development efficiency, achieve quick and easy process, becomes a stable and secure software systems.
\end{abstract}

\section{Introduction}

Computer cannot directly understand high-level language, can only understand the machine language, it is necessary to translate high-level language into machine language, so the computer can execute programs written in high-level languages. Thus producing the corresponding compiled languages available to programmers. There are two types translation languages, compiled and interpreted, the difference in two ways generally only translated at different times[1].

Compiled and interpreted, both have their advantages and disadvantages. The former is due to the speed of the program execution, the same conditions for the lower system requirements, so it could be used in the development of operating systems, large applications, database systems, etc.

In the aspect of data storage, traditional relational database, is facing the following problems: Performance High - demand for high concurrent read and write in database; Huge Storage - the demand for efficient storage and access of massive data; High Scalability \& High Availability-The high scalability and high availability of the database.

Compared with the traditional database, XML database can access and manage semi-structured data effectively, provide to the operation of the label and the path. In addition, the XML database can store and query the heterogeneous document structure, and provide support for the access to the heterogeneous information.

\section{Programming language}

Before the execution of the program, the compiler needs a special compiling procedure, and the program compiled into a machine language file, such as EXE file, later to run, then do not re-translated, compiled the results directly on the line (exe file).Because the translation only once, the runtime does not need to be translated, so a compiled language program execution efficiency is high.

The different explanations, the program does not require explanatory language compiler, saving procedure. Explanatory language when running the only translation, such as basic--interpretive language, there is a special explanation can perform basic program directly, each statement translation is performed before time. Such an interpreted language must be translated once each execution, efficiency is low relatively. Interpretation is the translation of a sentence[2]. 
However some of the web page script, server scripts, as well as secondary development interface for speed requirements is not high, there are some requirements for the compatibility between different systems, and the program is usually interpreted language,such as JavaScript, Java, VBScript, Perl, Python, Ruby, MATLAB, etc.

So in this case, if there is a combination of above two types of development patterns, not only can shorten the process of software development but also can make the software developed more efficient and more secure.

\section{Data storage}

Today, the development of database technology is changing with each passing day.The database can be divided into: relational database and XML database.The traditional relational database such as SqlServer/Orcale faces the following obstacles[3]:

High performance - the demand for High database concurrency, speaking, reading and writing.

Web2.0 website according to the user's personalized information to generate real-time dynamic pages and provide dynamic information,so basically, the use of dynamic page static technology, the database concurrency load is very high,often thousands of times per second read and write requests.A thousand times to cope with the relational database SQL query barely hold up, but to cope with tens of thousands of times a SQL request to write data, the hard disk IO had been unable to bear.

Huge Storage - the demand for efficient storage and access of massive data.

For a relational database, in a 250 million records list for SQL queries, the efficiency is very low and even intolerable. For example, the large web site the user login system, such as Tencent, Shanda, often hundreds of millions of accounts, a relational database is difficult to deal with.

\section{High Scalability \&High Availability-High scalability and high availability for database} needs.

In a Web-based architecture, the database is the most difficult scale[4]. When an application user and the views growing, your database has no way as easy as Web server, app server by adding more hardware and services nodes to scale performance and capacity. For many Web sites that offer 24-hour service, upgrades and extensions to database systems is a very painful thing, and often require downtime maintenance, and data migration.

\section{XMl database}

$\mathrm{XML}$ database is a data management system which supports the storage and query of XML format documents.In the system, the developer can query, export and specify the serialization of the XML documents in the database. Compared with the traditional database, XML database has the following advantages:

XML database allows for a semi-structured data access and management effectively.

Such as web content is a kind of semi-structured data, and traditional relational database for similar web content such semi-structured data can not be effectively managed.

Provide operation for labels and paths.

Traditional database language allows the data elements of value, not on names of elements operation, semi-structured database provides operation on the tag name, also includes the operation on the path.

The XML database is easy to operate the hierarchical data.

When the data itself has hierarchical features, because the XML data format can clearly express the level of data, so the XML database is easy to operate on hierarchical data.XML database for managing complex data structure of data sets.If you have stored information in XML format, the XML database is conducive to document storage and retrieva, and provide high-quality full-text search engine.In addition, the XML database can store and query the heterogeneous document structure, and provide support for the access to the heterogeneous information. 


\section{Transplant the "electronic patient" case editing module to different medical teaching or emergency system software.}

\section{The module steps are as follows:}

A:The system displays a case editing interface and accepts information inputs. Waiting for the user to operate (save, modify, delete) commands.

$\mathrm{B}$ :When the data storage operation is triggered, the system first carries on the data verification, the system internal XML database engine automatically generates the structure XML file,and initialization operation.

$\mathrm{C}$ :When the trigger data query or delete operation, the system first query criteria for data validation, after a check is successful, traversing through the query XML library, find the appropriate label, the data corresponding operations.

D:When the amount of data need backup, in order to data classification and security backup, take combined with relational databases, XML files by key value indexing directly stored in relational database. Extracting a segment data can be queried according to the key values[5].

\section{Concrete implementation:}

The structure of the XML is a data structure containing two groups of cases.

The data table field edited by the case is as follows:

Patient Content

1、Basic Info

Patient Name

Patient Height

Patient Sex

2、Patient State Editor

Pupil Diameter

ECG

Blood Pressure

Air Way ObSrtuction

3、 Patient Op Edito

Set Ari Way Open

Set Bron Chial Cannula

Set Defibllation Num

Set CarotidWave

XML data table structure instance:

$<$ ?xml version="1.0" encoding="gb2312"?>

$<$ Root $>$

$<$ PatientCase Name="CaseOne" $>$

$<$ PatientContent $>$

$<$ BasicInfo $>$

$<$ PatientName Value="Jackie" Unit="'"/>

$<$ PatientHeight Value="170" Unit="cm"/>

$<$ PatientSex Value="M" Unit="'/>

$<$ PatientWeight Value="80" Unit="kg" />

$<$ PatientAge Value="30" Unit="y" />

$</$ BasicInfo $>$

$<$ PatientStateEditor $>$

$<$ PupilDiameter Value="5" Unit="mm"/>

$<$ ECG Value="80" Unit="bmp"/>

$<$ BloodPressure Value="120/80" Unit="mmHg"/>

$<$ AirWayObSrtuction Value="Yes" Unit="'/>

$<$ Laryngismus Value="No" Unit="" /> 


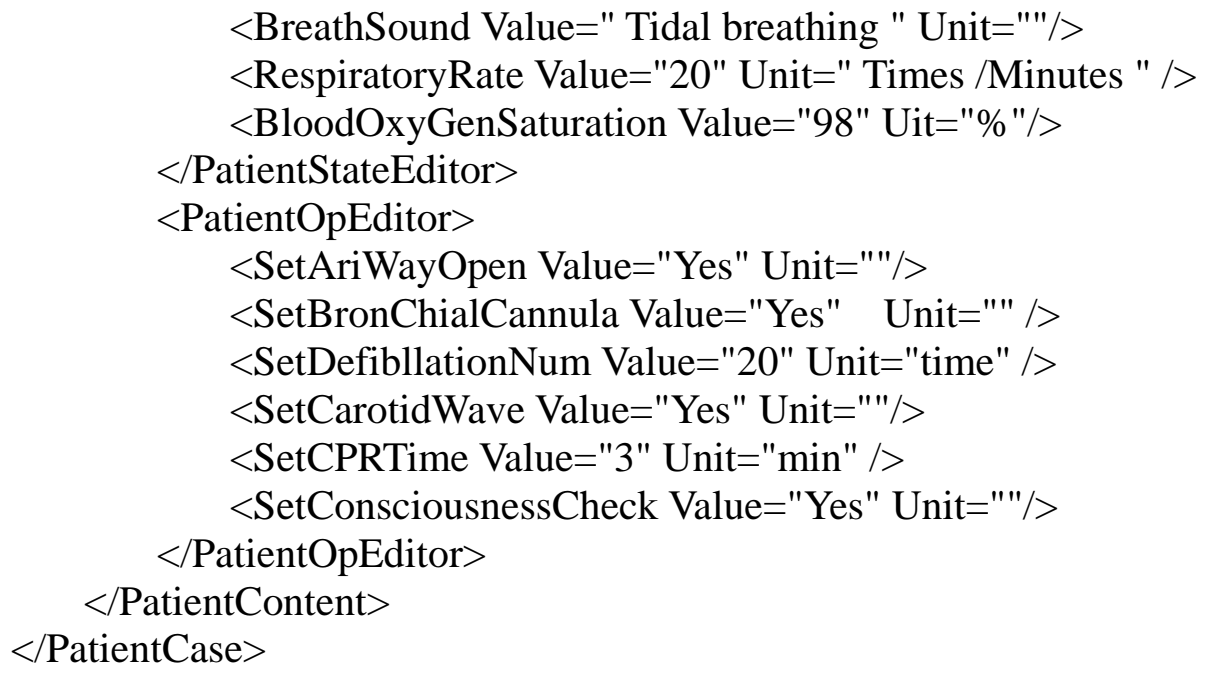

\section{Conclusion}

The above XML structure table can avoid the inconvenience caused by large database, and improve the portability of data.. With the modular software structure, when the software system appears big changes can be released through the module interface free transplant case editing module, and to ensure that the data will not be lost or damaged.

\section{Acknowledgement}

In this paper, the research was sponsored by the Twelve-Five comprehensive investment project(Project No. B01-0818)、 the 2012 Colleges and Universities in Tianjin Science and Technology Development Fund Program (Project No. 20120801) and Teaching Reform Project of Tianjin University of Technology(Project No. YB13-35).

\section{References}

[1] XU Jin-feng,ZHAO Wen-jing.XML and Relation DB-Based Distributed Common Workfolw Engine. [J]. MICROELECTRONICS \& COMPUTER ,2004.21(10).

[2] LIU Chen,CAI Chang-tao.Research on Technology of CAPP Data Description Based on XML[J].APPLICATION RESEARCH OF COMPUTERS,2005.22(4).

[3]WANG Zhi-ping .XML-Based Heterogeneous Relational Databases Integration System.[J].JOURNAL OF HENAN UNIVERSITY(NATURAL SCIENCE) ,2007.37(5).

[4]Florescu Dank KossmannD.Storing and querying XML data using an RDBMS,IEEE Data Engineer Bulletin,1999.22(3):27-34.

[5]H.Jiang,H.Lu,W Wang and J.X.Yu.Path materialization revisited:An efficient storage model for XML data.In Proc.OF ADC,2002. 